\section{Diagnosis of a Pleural \\ Mesothelioma by \\ Endosonography-Guided \\ Transgastric Fine-Needle Aspiration}

A 73-year-old man presented to a local hospital with chest pain and pleural effusion. Thoracentesis revealed a hemorrhagic exudate without malignant cells. Computed tomography showed a mass $5 \times 5 \mathrm{~cm}$ in size at the left diaphragm, extending into the peritoneal cavity adjacent to the stomach. On gastroscopy, a large mass was detected in the gastric fundus, with intact overlying mucosa (Figure 1). The patient was referred to our institution for further work-up. Endoscopic ultrasonography (EUS) using a curved-array echo endoscope (Pentax FG-34UX) revealed a lobulated hypoechoic mass compressing the intact gastric wall (Figure 2). The precise cranial extent of the mass could not be defined. There were no enlarged perigastric or celiac lymph nodes. EUS-guided transgastric fine-needle aspiration (FNA) was performed, and cytological examination of the aspirate showed numerous clusters of large atypical mesothelioma cells. The diagnosis was confirmed by positive immunohistochemistry staining for keratin (Figure 3) and negative staining for HEA 125. The patient was deemed to be inoperable; the tumor progressed despite palliative chemotherapy, and he died six months later.

Endosonography is a well-established method for distinguishing between submucosal gastric tumors and extraluminal compression [1]. Transgastric FNA allows accurate tissue diagnosis in most cases of extraluminal masses, with somewhat lower accuracy rates reported for submucosal masses [2]. Large EUS series for patients with "submucosal" gastric tumors have not reported mesotheliomas in the differential diagnosis $[1,3]$. As was the case in the

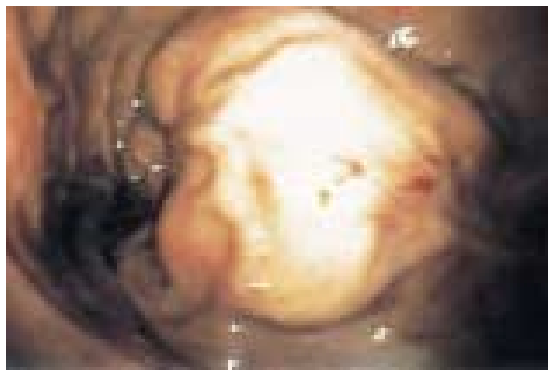

Figure 1 The endoscopic image showing an extrinsic mass compressing the gastric fundus. The overlying gastric mucosa appears intact

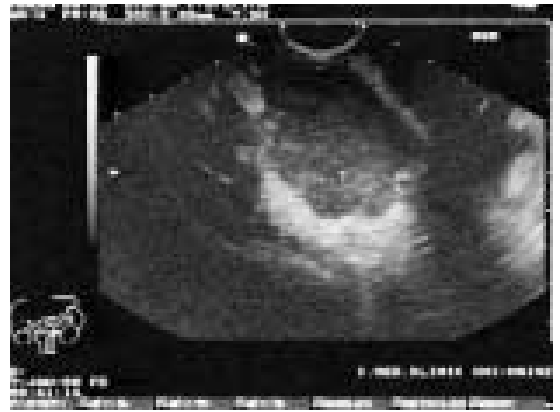

Figure 2 Endosonographic image of the lobulated hypoechoic lesion (T) adjacent to the gastric fundus

patient reported here, intra-abdominal growth of pleural mesotheliomas may occur, and this represents an advanced stage of disease (T4). EUS-guided FNA provides a low-risk approach for diagnosing such lesions.

H. Gerke ${ }^{1}$, F. Bittinger $^{2}$, P. R. Galle ${ }^{1}$, K. Mergener ${ }^{1}$

${ }^{1}$ First Dept. of Medicine, Johannes

Gutenberg University, Mainz, Germany

${ }^{2}$ Institute of Pathology, Johannes

Gutenberg University, Mainz, Germany

\section{References}

${ }^{1}$ Rösch T. Endoscopic ultrasonography in upper gastrointestinal submucosal tumors: a literature review. Gastrointest Endosc Clin N Am 1995; 5: 609-615

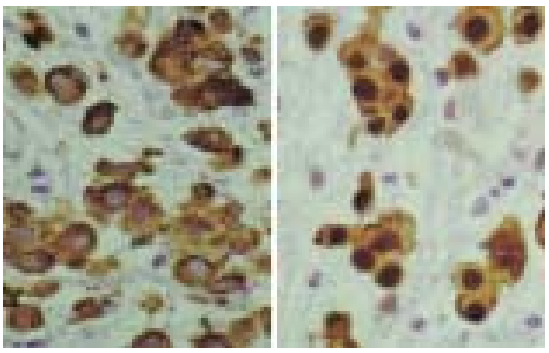

Figure 3 Fine-needle aspirate, with numerous clusters of large mesothelioma cells with a low nucleocytoplasmic ratio, anisonucleosis, and oc casional prominent nucleoli. The mesothelioma cells show a typical staining pattern, with strongly positive staining for keratin (mAb KL1) (left) and calretinin (right) (DAB, original magnification $\times 400$ )

${ }^{2}$ Wiersema MJ, Vilman P, Giovannini M, et al. Endosonography-guided fine-needle aspiration biopsy: diagnostic accuracy and complication assessment. Gastroenterology 1997; 112: 1087-1095

${ }^{3}$ Fritscher-Ravens A, Schirrow L, Atay Z, et al. Endosonographically controlled fine-needle aspiration cytology: indications and results in routine diagnosis. $\mathrm{Z}$ Gastroenterol 1999; 37: 343-351

Corresponding Author

K. Mergener, M.D.

Gastroenterology Section

Virginia Mason Medical Center P.O. Box 900

Seattle, WA 98111, USA

Fax: +1-206-223-6379

E-mail: gaskdm@vmmc.org 